\title{
Interferometrische Untersuchung der Deformation von Laserstäben während des Pumpvorganges
}

\author{
E. BAyer und G. SchaAcK \\ Institut für Technische Physik der Technischen Hochschule Darmstadt \\ (Z. Naturforschg. 21 a, 643-653 [1966] ; eingegangen am 7. Februar 1966)
}

\begin{abstract}
The elongation of neodymium-glass laser-rods (LG 52 and LG 55 of S c h ot t u. Gen., Mainz), the curvature and the tilting of their end surfaces have been measured by means of a MicheLsoninterferometer, a rotating-drum-camera and a laser illuminating the interferometer with short, isolated "spikes" during the pumping pulse of the rod. The pumping arrangements used for the tested rods are an elliptical cylinder and a rotational ellipsoid. A linear dependence of the observed deformation from the absorbed pumping energy is observed for this four-level material, so that the elongation of the rod and the curvature and the tilting angle of the surfaces can be expressed in terms of the pumping energy.

The deformation measured in the elliptical cylinder pumping arrangement is compared with the calculated density of the absorbed energy within a laser rod illuminated by such an elliptical mirror. The main features of the observations are represented by this calculation.
\end{abstract}

Zur Erklärung des impulsförmigen Emissionsablaufs der meisten optisch gepumpten Festkörperlaser geht man häufig von der Annahme aus, daß im starren Resonator mit idealer geometrischer Form (z. B. planparallele oder konfokale Anordnung) eine größere Zahl von Eigenschwingungen gleichzeitig angeregt ist, deren räumlich verschieden variierende Feldstärken eine nicht gleichmäßige Verteilung der Inversion im aktiven Medium hervorrufen. Die damit verknüpfte variable Verstärkung führt wiederum zu einem ständigen Wechsel der jeweils vorherrschenden Eigenschwingungen und ihrer Amplituden ${ }^{1,2}$. Dieses Bild ist nicht vollständig, denn es berücksichtigt nicht die mit dem Zünden der Blitzlampe einsetzende und während des Pumpvorganges fortdauernde Deformation des Resonators, die infolge Erwärmung des Laserstabes durch den nicht wieder als Strahlung emittierten Anteil der absorbierten Pumpenergie auftritt. Diese stetig zunehmende Deformation des Resonators führt ebenfalls zu einem Ablösen der momentan angeregten Eigen-. schwingungen durch solche, die bei einer späteren, stärker von den Ausgangsabmessungen abweichenden Resonatorform größere Verstärkung und geringere Verluste haben. Da aber schon geringfügige Änderungen der Verluste, d. h. Änderungen der Resonatorgüte von der Größenordnung $1 \%$ zum er-

1 C. L. Tang, H. Statz u. G. De Mars, J. Appl. Phys. 34, 2289 [1963].

2 H. Statz u. C. L. Tang, J. Appl. Phys. 35, 1377 [1964].

3 E. Bayer, K. H. Hellwege u. G. SchaAck, Z. Naturforschg. 20 a, 1181 [1965]. neuten Anfachen von Relaxationsschwingungen beträchtlicher Amplitude führen können ${ }^{3}$, muß mit einem erheblichen Einfluß dieser Deformation auf das Zustandekommen des unregelmäßigen Schwankens der emittierten Intensität gerechnet werden. $\mathrm{Da} \beta$ schon scheinbar geringfügige Abweichungen von der Idealform des Resonators zu erheblichen Güteminderungen und Änderungen in der Form der Eigenschwingung Anlaß geben können, zeigen beispielsweise die numerischen Rechnungen von Fox und $\mathrm{LI}^{4}$ für ebene, nicht parallele Spiegel. Experimentell wurden Änderungen der Schwingungsform während des Pumpimpulses durch Nahfeld-Beobachtungen mit Mitteln der Kurzzeitphotographie beispielsweise von Stickley ${ }^{5}$ untersucht, der fand, daß während eines Pumpimpulses mit fortschreitender Erwärmung des Stabes stets Eigenschwingungen einfacher Form durch solche komplizierter Feldverteilung abgelöst werden. Diese Beobachtung entspricht den Rechnungen von IKenoue et al. ${ }^{6}$, die zeigten, $\mathrm{da} ß$ in einem Laserresonator mit nicht exakt planparallelen Endflächen bei großen Fresnel-Zahlen Eigenschwingungen höherer Ordnung kleinere Verluste haben als beispielsweise die longitudinale Grundschwingung.

In jüngster Zeit ist in einer Reihe von Untersuchungen die Existenz derartiger Deformationen nach-

4 A. G. Fox u. T. Li, Proc. I.E.E.E 51, 80 [1963].

5 C. M. Stickley, Appl. Opt. 2, 855 [1963].

6 H. Ogura, Y. Yoshida u. J. Ikenoue, J. Phys. Soc. Japan 20, 598 [1965]. 
gewiesen worden ${ }^{7-15}$. Die quantitativen Angaben über Art und Größe der Deformation sind aber bisher unzureichend. Es ist deshalb das Ziel der vorliegenden Untersuchung, für einige spezielle Fälle solche quantitativen Aussagen zu liefern, die als Unterlage für eine genauere Berechnung von Form und Verlusten der Eigenschwingungen im deformierten Resonator dienen können.

\section{Experimentelles}

Die zur Messung verwendete Anordnung ist in Abb. 1 schematisch dargestellt. Der zu untersuchende Laserstab ist zusammen mit der Anordnung zum Pumpen in einem Arm des Michelson-Interferometers untergebracht. Im anderen Arm ist ein ungepumpter, aber sonst gleicher Laserstab zur Kompensation von Absorptions-, Reflexions- und Streuverlusten angebracht. Die Stirnflächen beider Stäbe sind für $\lambda=6943 \AA$ entspiegelt. Gegenüber anderen Interferometern hat das $\mathrm{MI}_{\mathrm{r}}$ CHELSON-Interferometer hier den Vorteil der verdoppelten Empfindlichkeit, da der Stab zweimal von der Meßstrahlung durchlaufen wird.

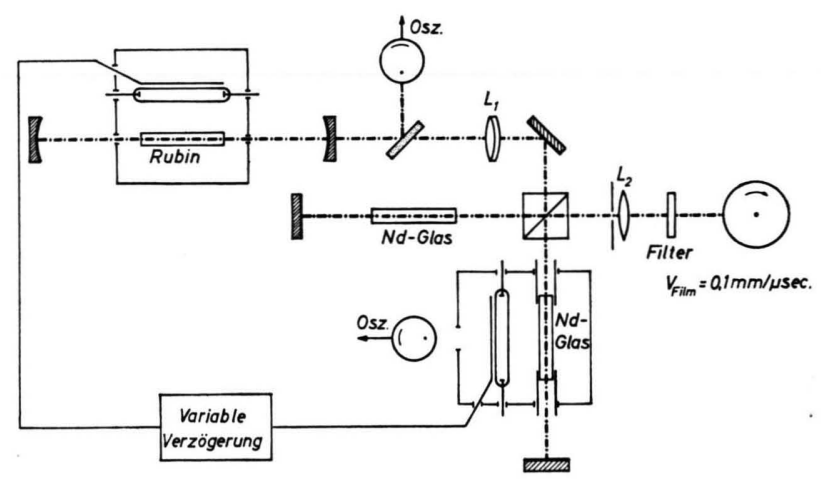

Abb. 1. Schema der Meßanordnung. Links: Beleuchtungslaser, Mitte: Michelson-Interferometer, Rechts: Trommelkamera. Linse L 1 sammelt das vom Beleuchtungslaser emittierte divergente Bündel, so daß es gerade den Querschnitt des Untersuchungsobjekts ausfüllt. Filter: S c h o t t RG 5, 2 mm dick. Linse L 2 entwirft die von Interferenzen gleicher Dicke durchzogenen Bilder der Stäbe in der Filmebene der Trommelkamera. Filmmaterial: K o d a k - Tri-X-Pan.

Zur Beleuchtung des Interferometers mit monochromatischem, interferenzfähigem Licht dient, ähnlich wie in ${ }^{14}$, ein Rubinlaser mit zwei konkaven Außenspiegeln von $1 \mathrm{~m}$ Krümmungsradius in $1,70 \mathrm{~m}$ Abstand. Die

7 U. J. Schmidt, J. Appl. Phys. 35, 259 [1964].

8 A. E. Blume u. K. F. Tittel, Appl. Opt. 3, 527 [1964].

9 Y. A. Ananew u. A. A. MaK, Opt. Spectr. USSR 19, 578 [1964].

10 E. Bayer, K. H. Hellwege u. G. Schaack, Phys. Verhandlg. DPG 1964, S. 296.

11 H. G. HäFELE, Z. Naturforschg. 19 a, 1353 [1964].
Emission dieses Lasers besteht bei passend gewählter Pumpleistung aus sehr schmalen, intensiven Impulsen ohne kontinuierlichen Untergrund und dauert etwa 800 bis $1000 \mu \mathrm{s}$. Der einzelne Impuls hat eine Halbwertszeit von $5 \mu \mathrm{s}$ im Mittel. Zu Beginn der Emission folgen die „spikes“ in Zeitabständen von etwa $10 \mu$ s nacheinander, in der zweiten Hälfte verlängert sich dieser Abstand auf etwa $30-50 \mu$ s. Diese letztgenannte Zeitspanne ist groß genug, um auf einer Kamera mit rotierender Filmtrommel (Filmgeschwindigkeit ca. $100 \mathrm{~ms}^{-1}$ ) getrennte Interferenzbilder von etwa $5 \mathrm{~mm}$ Durchmesser ohne störende Bewegungsunschärfe zu erzeugen. Die Emission des Beleuchtungslasers und der Verlauf der Pumpstrahlung im Interferometer werden auf einem Zweistrahloszillographen registriert, so daß die einzelnen Interferenzbilder auf einer Zeitskala, beginnend mit dem Einsatz der Pumpstrahlung, mit einer Genauigkeit von etwa $\pm 10 \mu$ s eingeordnet werden können. Mit einer elektronischen Verzögerungseinheit kann die Zündung der Blitzlampe im Beleuchtungslaser beliebig gegen den Pumpimpuls im Interferometer verschoben werden.

Es wurden zwei Pumpanordnungen untersucht:

a) Ein elliptischer Zylinder (s. Abb. 1) mit den Abmessungen: große Achse $a=5 \mathrm{~cm}$, kleine Achse $b$ $=4,35 \mathrm{~cm}$, Höhe $h=8 \mathrm{~cm}$. Hier war das Meßobjekt an beiden Enden mit je etwa $2 \mathrm{~mm}$ Länge in zwei Metallrohren eingespannt, die ihrerseits starr mit den Stirnflächen des elliptischen Zylinders verbunden waren und den Lichtweg zwischen Stab und Interferometerspiegel sowie zwischen Stab und Teilerwürfel vor Bestrahlung durch die Blitzlampen schützten.

b) Ein Rotationsellipsoid nach Röss ${ }^{16}$, bei dem Lampe und Laserstab auf der Rotationsachse jeweils zwischen Brenn- und Scheitelpunkt liegen, mit den Abmessungen: große (Rotations-) Achse $a=8,0 \mathrm{~cm}$, kleine Achse $b=7,45 \mathrm{~cm}$. Hier war der Laserstab im Brennpunkt des Ellipsoids starr eingespannt, das andere Ende war in axialer Richtung beweglich, in radialer Richtung geführt. Der Interferometerspiegel im Meßarm war ersetzt durch eine Aluminium-Verspiegelung auf der unteren festen Stirnfläche des Meßstabes.

Zur Kontrolle der mechanischen Stabilität des Interferometers wurde das Meßobjekt durch ein Metallrohr ersetzt, so daß die Streifensysteme des Interferometers unter sonst gleichen Bedingungen photographiert werden konnten. Es zeigte sich, daß bis zu einem Zeitpunkt $600 \mu$ s nach dem Zünden der Blitzlampe im Pumpgehäuse das Interferometer völlig starr war. $700 \mu$ s nach der Zündung hatte die durch die Entladung ausgelöste mechanische Deformation des Interferometers eine Streifenverschiebung von ein Achtel Streifenbreite

12 A. P. Veduta, A. M. Leontowich u. V. N. Smorchrow, Soviet Phys.-JETP 21, 59 [1965].

13 D. White u. D. Gregy, Appl. Opt. 4, 1034 [1965].

14 R. L. Townsend, C. M. Stickley u. A. D. Maio, Appl. Phys. Letters 7, 94 [1965].

15 H. Welling, C.J. Bickart u. H.G. Andresen, I.E.E.E. Journ. Quantum Electronics 1, 223 [1965].

16 D. Röss, Appl. Opt. 3, 259 [1964]. 
erreicht. Diese Verschiebung blieb aber für mindestens weitere $500 \mu$ s bei vorgegebener Pumpanordnung und Pumpleistung völlig reproduzierbar, wie wiederholte photographische Aufnahmen und Kontrollmessungen mit einem piezoelektrischen Mikrophon gezeigt haben. Da aber die Emission der untersuchten Laserstäbe bei entsprechender Verspiegelung der Endflächen nur in einer Zeitspanne zwischen etwa $200 \mu$ s und $800 \mu$ s nach der Zündung erfolgt, kann die während der induzierten Emission auftretende Verformung des Stabes ohne Störung durch Schwingungen des Interferometers beobachtet oder (gegen Ende des Emissionsvorganges) nach Anbringen einer entsprechenden Korrektur bestimmt werden. Nach etwa $10-20 \mathrm{~ms}$ sind diese Schwingungen wieder soweit abgeklungen, daß auch die im Vergleich zum Erwärmungsvorgang langsam erfolgende Abkühlung des Laserstabes störungsfrei beobachtet werden kann.

\section{Interferogramme}

Wegen ihrer hohen optischen Homogenität wurden vorwiegend Laserstäbe aus Neodym-dotiertem Glas untersucht, und zwar im elliptischen Zylinder die Gläser LG 52 und LG 55, im Rotationsellipsoid das Glas LG 55 der Firma Jenaer Glaswerk S c h o t t u. Gen., Mainz. Die Stäbe hatten eine Länge von $70 \mathrm{~mm}$, eine bestrahlte Länge $l=65 \mathrm{~mm}$ und einen Durchmesser von $7 \mathrm{~mm}$. Die Mantelflächen waren poliert. Die für das folgende wichtigen Eigenschaften der beiden Glassorten sind in Tab. 1 zusammengestellt.

\begin{tabular}{|c|c|c|}
\hline Bezeichnung & LG 52 & LG 55 \\
\hline Grundglas & $\begin{array}{l}\text { Borosilikat- } \\
\text { glas }\end{array}$ & Silikatglas \\
\hline Dotierung & $2 \% \mathrm{Nd}_{2} \mathrm{O}_{3}$ & $5 \% \mathrm{Nd}_{2} \mathrm{O}_{3}$ \\
\hline Brechungsindex $n_{\mathrm{D}}$ & 1,669 & 1,516 \\
\hline $\begin{array}{l}\text { Ausdehnungskoeffizient } \\
\quad(1 / l) \cdot(\mathrm{d} l / \mathrm{d} T) \text { in }\left({ }^{\circ} \mathrm{C}\right)^{-1}\end{array}$ & $7,8 \cdot 10^{-6}$ & $10,7 \cdot 10^{-6}$ \\
\hline $\begin{array}{l}\mathrm{d} n / \mathrm{d} T \text { (Vakuum) für } \\
\quad \lambda=7000 \AA \text { in }\left({ }^{\circ} \mathrm{C}\right)^{-1}\end{array}$ & $2,6 \cdot 10^{-6}$ & $-1,6 \cdot 10^{-6}$ \\
\hline
\end{tabular}

Tab. 1. Eigenschaften der Neodym-Gläser LG 52 und LG 55 (nach Angaben des Herstellers Schott u. Gen.) .

Es wurden auch Rubinproben untersucht, jedoch hatten die uns zur Verfügung stehenden Stäbe keine genügende Homogenität, um Auswertungen mit einer Genauigkeit wie beim Neodymglas zuzulassen. Die von uns beobachteten Effekte sind vergleichbar mit den von Veduta et al. ${ }^{12}$ beschriebenen Beobachtungen.
Für die Genauigkeit, mit der die Änderungen der optischen Weglänge beim Pumpvorgang angegeben werden können, ist die photographische Qualität der Interferogramme maßgebend, die durch zwei Störeinflüsse herabgemindert wird:

1. Die Intensität des beleuchtenden Laserlichts ist nicht konstant über den Bündelquerschnitt, sondern zeigt die für einen Rubinlaser mittlerer Kristallqualität typische Struktur, die zu einer starken Körnigkeit des photographierten Bildes führt. Wegen dieser Körnigkeit hat es sich als günstig erwiesen, bei der Justierung der Interferometerspiegel diese nicht exakt senkrecht, sondern mit einem kleinen Keilwinkel zueinander einzustellen, so daß der Kristallquerschnitt vor dem Pumpvorgang von einigen parallelen Streifen durchzogen wird. Wir fanden eine Zahl von 3 bis 5 Streifen optimal. Eine größere Streifenzahl ist wegen der beschriebenen Körnigkeit nicht immer klar aufzulösen, auch wird die Streifenkrümmung und -verschiebung und damit die Nachweisempfindlichkeit bei gegebener Wölbung geringer. Eine kleinere Streifenzahl führt dagegen an den Wölbungsmaxima zu sehr breiten und stark gekrümmten Streifen, bei denen Schwärzungsmaxima und -minima nicht genügend genau lokalisiert werden können.

2. Die Bewegungsunschärfe beträgt in Anbetracht der genannten Daten über Filmgeschwindigkeit und Halbwertsbreite eines „spikes“ etwa 0,5 mm. Ihr Einfluß kann herabgesetzt werden, indem man die Interferometerspiegel so justiert, daß die Interferenzstreifen parallel zur Filmtransportrichtung liegen. Auch aus diesem Grunde ist es also zweckmäßig, eine starke Krümmung der Streifen zu vermeiden.

In Abb. 2 sind zusammen mit dem zeitlichen Verlauf der Pumpleistung einige mit der Anordnung gewonnene Interferenzaufnahmen für die verschiedenen Pumpanordnungen und Glassorten wiedergegeben. Abb. $2 \mathrm{~d}$ zeigt Interferogramme, die bei absichtlicher Fehljustierung der Lampe im elliptischen Zylinder erhalten wurden. Die Lampe war dabei aus der Brennlinie um den Lampenradius in die Richtung des Ellipsenmittelpunktes verschoben worden.

Die Richtung der Streifenwanderung nach dem Zünden der Blitzlampe und die sich allmählich verstärkende Krümmung dieser Streifen zeigen, daß die optische Weglänge $n \cdot l$ im Kern des Stabes stärker zunimmt als am Rand. Wenn man annimmt, daß die Zunahme der optischen Weglänge homogen 


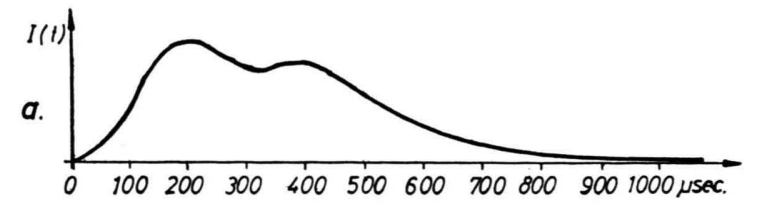

b.

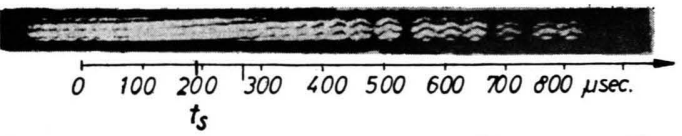

c.

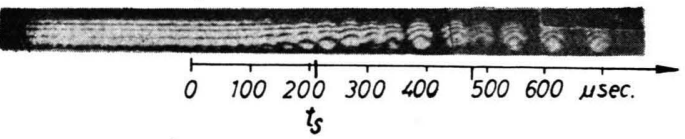

d.

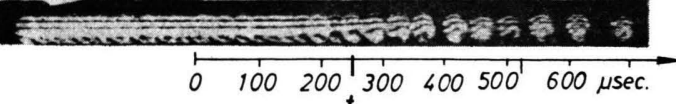

e.

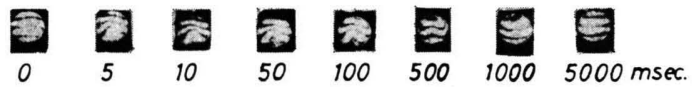

Abb. 2. Mit der in Abb. 1 dargestellten Anordnung erhaltene Interferogramme. a) Verlauf der Intensität des Pumplichts. b) LG 55 im elliptischen Zylinder. Der Zeitmaßstab kennzeichnet den Verlauf des Pumpimpulses. Die Blitzlampe wird bei $t=0$ gezündet, bei $t=t_{\mathrm{s}}$ ist der Schwellenwert für den Einsatz der induzierten Emission erreicht. c) LG 52 im elliptischen Zylinder. d) LG 52 im elliptischen Zylinder bei fehljustierter Lampe. e) Ausgleich des Temperaturgefälles in radialer Richtung nach Beendigung des Pumpimpulses im elliptischen Zylinder. Aufnahme bei ruhender Filmtrommel. Bei allen Bildern ist die Blitzlampe auf der rechten Seite zu denken.

längs der Stabachse verteilt ist, dann kann man den Effekt am einfachsten durch eine vom Stabinneren aus gesehen konkave Wölbung der Stabendflächen beschreiben ${ }^{14}$, wobei zu der mechanisch meßbaren Wölbung dieser Endflächen noch der Einfluß der Temperaturabhängigkeit des Brechungsindex tritt. Eine solche konkave Wölbung der Stabendflächen steht im Einklang mit den verschiedenen Berechnungen der Energieverteilung in einem gepumpten Laserstab (z. B. ${ }^{17}$ ). Die Stärke dieser Krümmung ist abhängig von Glassorte und Pumpanordnung. Es zeigt sich ferner, daß beim Pumpen im elliptischen Zylinder neben der Krümmung der Resonatorflächen auch eine Neigung infolge der ungleichmäßigen Ausleuchtung (s. u.) durch die Blitzlampe eintritt, die im Interferogramm in einem Anheben der Streifen auf der der Lampe zugewandten Seite des Meßstabes erkennbar wird. Besonders deutlich ist dieser Effekt in Abb. 2 d, wo die Verbiegung des Stabes durch die Fehljustierung der Lampe verstärkt wird.

17 G. E. Devlin, J. McKenna, A. D. May u. A. L. Schawlow, Appl. Opt. 1, 11 [1962].
Die Lampe war dabei aus der Brennlinie in Richtung auf den Ellipsenmittelpunkt um eine Strecke von der Größe des Lampenradius verschoben.

Abb. 2 e zeigt Aufnahmen, die bei ruhender Filmtrommel entstanden sind und die die Vorgänge im Stab nach dem Abklingen des Pumpimpulses zeigen, nämlich das Aufheizen der Randschichten des Stabes durch die vom Lampenmantel ausgehende Wärmestrahlung. Die ursprünglich vorhandene konkave Krümmung wechselt infolge dieser Aufheizung nach einiger Zeit in eine konvexe Krümmung. Nach rund $10 \mathrm{~s}$ ist auch diese Strahlung abgeklungen und der Temperaturausgleich in radialer Richtung praktisch erreicht. Die am Wandern der Interferenzstreifen verfolgbare Abkühlung des Stabes auf Umgebungstemperatur dauert dagegen mehrere Minuten.

Zur Bestimmung der Änderung $\Delta[(n-1) l]$ der optischen Weglänge des Stabes im Interferometer wurde das zur Zeit $t$ erhaltene Interferogramm mit einem vor dem Zünden der Blitzlampe zur Zeit $\boldsymbol{t}_{\mathbf{0}}$ photographierten Interferogramm verglichen und die Zahl $z$ der in $\left(t-t_{0}\right)$ vorbeigewanderten Streifen

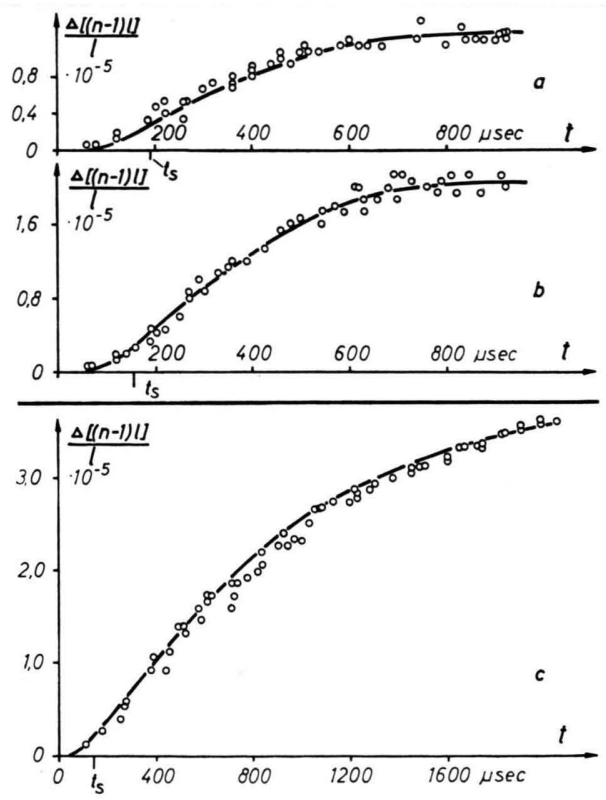

Abb. 3. Zunahme der relativen optischen Weglänge in der Achse eines Laserresonators mit festen Außenspiegeln während des Pumpimpulses. Die durchgezogene Kurve ist das Integral (2) über die von der Blitzlampe abgestrahlte Leistung in relativen Einheiten, die in einem Punkte an die Meßpunkte angeglichen ist. a) LG 55 im elliptischen Zylinder; b) LG 52 im elliptischen Zylinder; c) LG 55 im Rotationsellipsoid. Hier hat der Verlauf von $I(t)$ eine andere Form als in Abb. 2 a dargestellt. $t_{\mathrm{S}}$ kennzeichnet den Einsatz der induzierten Emission in dem in Abschn. 2 beschriebenen Resonator. 
einschließlich der Bruchteile bestimmt. Dann ist $\Delta[(n-1) l]=\frac{1}{2} z \lambda_{0}$ mit $\lambda_{0}=6943 \AA$. Zur genauen Bestimmung der Streifenbruchteile wurden die Interferogramme mit Hilfe eines Kleinbildprojektors vergrößert aufgezeichnet. Bei sorgfältiger Auswertung läßt sich eine Genauigkeit von $\pm \lambda_{0} / 16$ für $\Delta[(n-1) l]$ erreichen.

Die Schwellenwerte der Laserstäbe für den Einsatz der induzierten Emision wurden unter den gleichen Pumpbedingungen wie bei den interferometrischen Messungen im elliptischen Zylinder bestimmt. Der Resonator bestand aus zwei ebenen, dielektrisch verspiegelten Platten $\left(R_{1}=99 \%, R_{2}=80 \%\right)$ im $\mathrm{Ab}$ stand von $35 \mathrm{~cm}$. Da mit dem Rotationsellipsoid der Schwellenwert eines derartigen Resonators nicht zu bestimmen ist, wurden alle Angaben für diese Pumpanordnung auf eine Pumpleistung bezogen, die die gleiche Zunahme $\Delta[(n-1) l]$ in der Stabachse bewirkte, wie sie im elliptischen Zylinder am Schwellenwert gemessen wurde.

\section{Ergebnisse}

In Abb. 3 ist die aus einer Anzahl von Interferogrammen bestimmte Zunahme der optischen Weglänge in der Achse des Laserstabes über der Zeit für die beiden Glassorten und Pumpanordnungen aufgetragen. Will man die Zunahme der optischen Weglänge $\Delta[n l]$ in einem Resonator mit Spiegeln auf den Stirnflächen des Stabes berechnen, so erhält man

$$
\Delta[n l]=\frac{(\mathrm{d} n / \mathrm{d} T)+n(\mathrm{l} / l) \cdot(\mathrm{d} l / \mathrm{d} T)}{(\mathrm{d} n / \mathrm{d} T)+(n-1)(1 / l) \cdot(\mathrm{d} l / \mathrm{d} T)} \Delta[(n-1) l] ;
$$

mit den Angaben aus Tab. 1 folgt für den Proportionalitätsfaktor der Wert 2,0 für LG 52, der Wert 3,7 für LG 55. Die eingezeichneten Kurven sind die aus Oszillographenaufnahmen der Pumpintensität $I\left(t^{\prime}\right)$ durch graphische Integration ermittelten Werte für die von der Blitzlampe abgestrahlte Energie:

$$
P(t)=\int_{0}^{t} I\left(t^{\prime}\right) \mathrm{d} t^{\prime} .
$$

Diese Kurven wurden im horizontalen (späten) Teil für einen Zeitpunkt $t$ an das Mittel der Meßpunkte in diesem Bereich angepaßt. Man sieht, daß insgesamt die Kurve $P(t)$ den Verlauf der Meßpunkte recht gut wiedergibt. Man kann daraus schließen, da $\beta$ der Anteil der in dem Stab absorbierten Leistung an der insgesamt von der Lampe emittierten
Lichtleistung immer konstant bleibt, d. h. daß die Absorptionseigenschaften des Lasermaterials während des Lampenimpulses praktisch konstant bleiben, wie man es für ein Vierniveausystem erwartet. Demzufolge ist also die Zunahme der optischen Weglänge des Laserstabes ein $\mathrm{Ma} ß$ für die von ihm absorbierte Pumpenergie. Sättigungseffekte, wie sie beim Rubin beobachtet werden, spielen hier keine Rolle. Außerdem folgt daraus, daß die Abklingzeiten der meisten angeregten Niveaus der $\mathrm{Nd}^{3+}$-Ionen in der Glasmatrix kürzer als etwa $100 \mu$ s sein müssen.
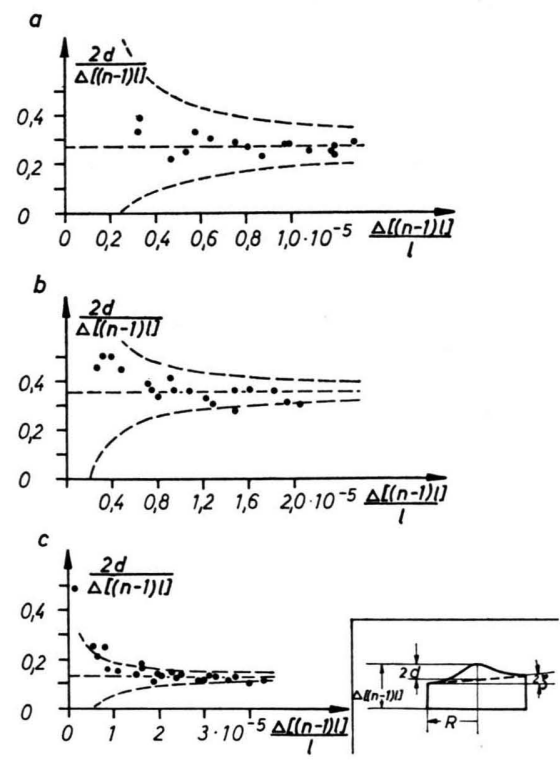

Abb. 4. Verhältnis der Auswölbung $2 d$ eines Resonators in der Ebene der Stabachse und der $\boldsymbol{a}$-Achse des Pumpgehäuses zur Zunahme der optischen Weglänge in der Stabachse $\Delta[(n-1) l]$. Die genaue Bedeutung der Bezeichnungen ist in der Skizze, die die Zunahme der optischen Weglänge in einem Resonatorquerschnitt darstellen soll, angegeben. Eingezeich. net sind gestrichelt die Ausgleichsgerade durch die Meßpunkte und die Schranken der Meßfehler. a) LG 55 im elliptischen Zylinder; b) LG 52 im elliptischen Zylinder; c) LG 55 im Rotationsellipsoid.

Entsprechend dieser Beobachtung sollte auch die Deformation des Laserstabes (Wölbung und Neigung der Endflächen) linear von der im Stab absorbierten Energie abhängen. In Abb. 4 ist das Verhältnis von scheinbarer Auswölbung des Resonators (in einem Schnitt längs der $a$-Achse für die Messungen im elliptischen Zylinder) zur Längenausdehnung des Resonators über dieser Längenausdehnung aufgetragen. Die genàue Bedeutung der Größen $d$ und $\Delta$ ist aus der Skizze ersichtlich, in der über einem 
Stabquerschnitt die Zunahme der optischen Weglänge aufgetragen ist. Die angegebenen Fehlerschranken sind berechnet aus einer Unsicherheit der Bestimmung von $d$ und $\Delta$ jeweils um $\lambda_{0} / 16$ in entgegengesetzter Richtung. Man erkennt, daß die Meßpunkte in allen Fällen gut auf einer horizontalen Geraden liegen.
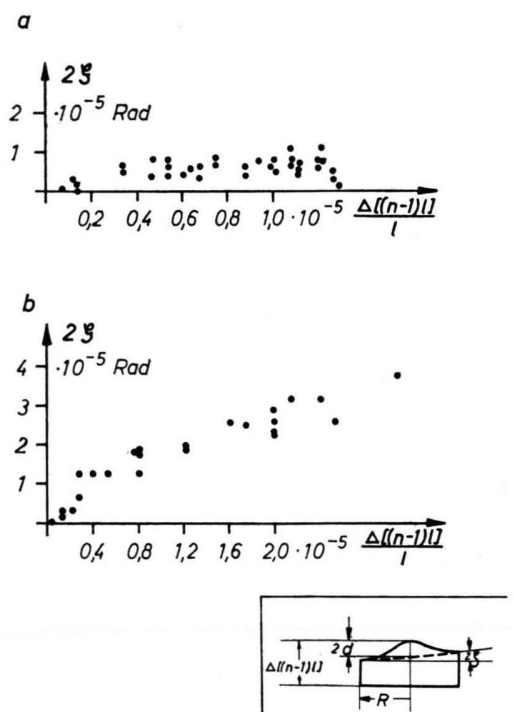

Abb. 5. Neigung $\zeta$ der Endflächen von Laserresonatoren beim Pumpen im elliptischen Zylinder. Die Bedeutung von $\zeta$ geht aus der Skizze hervor. Die gezeichnete Schnittebene ist die Ebene der Stabachse und der $a$-Achse des elliptischen Zylinders. a) LG 55; b) LG 52, beide im elliptischen Zylinder. Die $\zeta$-Werte erhöhen sich um die in Abschn. 3 angegebenen Faktoren für Stäbe mit aufgedampften Verspiegelungen.

In Abb. 5 ist ein $\mathrm{Ma} \zeta$ (siehe Skizze) für die Neigung einer Endfläche des Stabes ebenfalls über der Längsausdehnung des Resonators aufgetragen. Man erkennt auch hier eine Zunahme der Neigung mit der Pumpenergie, allerdings bildet eine durch den Nullpunkt gehende Gerade keine optimale Annäherung an die Meßpunkte, sondern die Neigung scheint zu Beginn des Pumpimpulses, d. h. bei kleinem $\Delta$, stärker anzuwachsen als zu einem späteren Zeitpunkt. Zur Erklärung dieser Abweichung scheidet ein Wärmeausgleich im Stabe wegen der Kürze der in Betracht kommenden Zeitspannen aus, wie eine Abschätzung zeigt. Dagegen kann eine systematische Fehlmessung anfänglich kleiner Neigung durch Überbewerten des kleinen beobachteten Effekts nicht ausgeschlossen werden. Im Rotationsellipsoid wird erwartungsgemäß keine Verbiegúng des Stabes beobachtet.

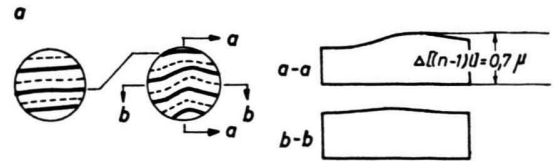

$b$

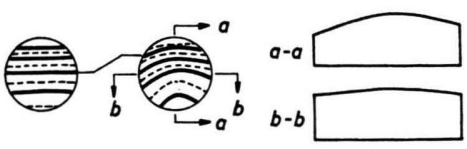

c

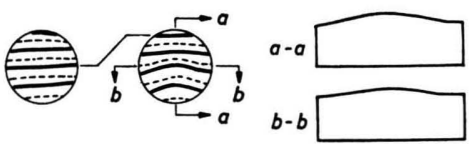

Abb. 6. Zunahme der optischen Weglänge in gepumpten Laserresonatoren. Die gezeichneten Schnitte liegen parallel zur $a$ und $b$-Achse des Pumpgehäuses. Gezeichnet sind ferner die Interferogramme vor dem Zünden der Blitzlampe und zu einem Zeitpunkt während des Impulses, wo die Zunahme der gesamten optischen Weglänge im Resonator gerade $0,7 \mu$ betrug. a) LG 55, elliptischer Zylinder; b) LG 52, elliptischer Zylinder; c) LG 55, Rotationsellipsoid.

Die linearen Beziehungen zwischen Pumpenergie, Längenausdehnung des Stabes sowie Deformation der Endflächen beim Neodymglas haben zur Folge, daß die Formen der Endflächen eines Stabes mit vorgegebener Pumpanordnung bei verschiedenen Pumpleistungen oder zu verschiedenen Zeitpunkten nach der Zündung der Blitzlampe stets einander ähnlich sind. Ebenso sind sie unabhängig von Form und Breite des Pumpimpulses, für die Deformation zum Zeitpunkt $t$ ist allein die bis dahin von der Lampe abgestrahlte Gesamtenergie $P(t)$ maßgebend. Zur Beschreibung der gemessenen Deformation können wir uns also auf die Angabe eines Bildes beschränken, aus dem alle anderen Formen durch Maßstabsänderungen herleitbar sind. In Abb. 6 ist in zwei Schnittebenen parallel zur $a$ - und $b$-Achse im elliptischen Zylinder die Zunahme der optischen Weglänge für LG 52 und LG 55 dargestellt, für das Rotationsellipsoid sind ebenfalls zwei senkrecht zueinander orientierte Schnitte gezeichnet (LG 55). Ferner sind in allen Fällen die Interferenzbilder, aus denen die Schnitte konstruiert wurden und die vor dem Zünden der Blitzlampe aufgenommenen Referenzbilder gezeichnet. Die Bilder sind so ausgewählt, daß gerade $\Delta[(n-1) l]=\lambda_{0}$ erfüllt ist. Die Deformation ist erwartungsgemäß drehsymmetrisch im Rotationsellipsoid, im elliptischen Zylinder ist sie 
symmetrisch bezüglich der großen Ellipsenachse. In dieser Richtung ist die Wölbung ausgeprägter als in der dazu senkrechten Richtung. Ferner erkennt man hier deutlich die Neigung der Endflächen um eine Achse parallel $b$, die dazu führt, daß der Bereich maximaler Ausdehnung außerhalb der Stabachse liegt und zur Lampe hin verschoben ist. Linien konstanter optischer Weglänge (Höhenlinien) hätten, im Stabquerschnitt eingezeichnet, die Form von Ovalen, deren große Achsen parallel $b$ außerhalb der Stabachse liegen.

Die beiden Glassorten LG 52 und LG 55 zeigen in der gleichen Pumpanordnung verschiedenes Verhalten. Bei LG 52 sind die Deformationen bei gleicher Pumpenergie deutlich stärker als bei LG 55, weil bei der zweiten Glassorte die Temperaturabhängigkeit des Brechungsindex $\mathrm{d} n / \mathrm{d} T$ negativ ist (Tab. 1) und die thermische Ausdehnung teilweise kompensiert. Auffällig ist ferner, daß die Krümmung der Stabenden bei LG 52 einsinnig ist, in den Schnitten durch die deformierten LG 55-Stäbe aber Wendepunkte auftreten. Dieser Unterschied liegt im größeren Absorptionsvermögen speziell im nahen UV und im größeren Brechungsindex von LG 52 begründet (s. u.). Vergleicht man die mit demselben Stab (LG 55) in beiden Pumpanordnungen gewonnenen Ergebnisse, so findet man eine kleinere Wölbung im Rotationsellipsoid nach Röss als im elliptischen $\mathrm{Zy-}$ linder. Im Rotationsellipsoid fehlt die für einen Laserresonator besonders schädliche Verbiegung des Stabes und die Verteilung der Energie über den Querschnitt ist gleichmäßiger, weil wegen des vorwiegend schräg zur Stabachse gerichteten PumplichtEinfalls im Glas größere Absorptionswege zurückgelegt werden und damit in den Randschichten des Stabes mehr Leistung absorbiert wird als beim elliptischen Zylinder. Die aus den Schnitten in Abb. 6 ermittelten Krümmungsradien sind in Tab. 2 aufgeführt. Sie gelten am Schwellenwert eines $7 \mathrm{~cm}$ langen
Stabes im elliptischen Zylinder oder bei der dazu äquivalenten Pumpleistung im Rotationsellipsoid (s. Abschn. 2).

Die Krümmungsradien sollten beim Pumpen im Rotationsellipsoid nicht von der Lage der Schnittebene durch den Stab abhängen. Die Differenz der beiden Wertepaare in der ersten Zeile von Tab. 2 gibt demnach einen Anhalt für die erreichbare Genauigkeit.

Aus der beobachteten Zunahme der optischen Weglänge

$$
\Delta[(n-1) \cdot l]=z \cdot \lambda_{0} / 2
$$

während des Pumpvorganges kann direkt die Erwärmung des Stabes bestimmt werden:

$$
\Delta T=\frac{z \cdot \lambda_{0} / 2}{l\{(\mathrm{~d} n / \mathrm{d} T)+(n-1) \cdot(1 / l) \cdot(\mathrm{d} l / \mathrm{d} T)\}} .
$$

Die Schnitte durch die Verteilung der optischen Weglänge über den Stabquerschnitt in Abb. 6 können also auch als Schnitte durch die Temperaturverteilung angesehen werden. Mit den Angaben in Tab. 1 und in Abb. 3 kann die Temperaturerhöhung in der Stabachse am Schwellenwert bestimmt werden. Die Ergebnisse sind ebenfalls in Tab. 2 eingetragen. Ein im elliptischen Zylinder gepumpter Rubinstab zeigt zum Vergleich am Schwellenwert eine Temperaturerhöhung ${ }^{11}$ von $1,1^{\circ} \mathrm{C}$.

\section{Berechnung der pro Volumelement absorbier- ten Leistung in einem Neodymglaslaser mit elliptischer Pumpanordnung}

In diesem Abschnitt sollen die oben dargestellten Wölbungen und Neigungen der Stabendflächen beim Pumpen im elliptischen Zylinder verglichen werden mit den Ergebnissen einer vereinfachten Berechnung der Dichte der im Stab absorbierten Leistung. Es wird dabei angenommen, daß die pro Volumelement im Stab freigewordene Wärme und damit die Än-

\begin{tabular}{|c|c|c|c|c|c|c|}
\hline \multirow{2}{*}{ Glasart } & Pumpanordnung & \multicolumn{2}{|c|}{ Krümmungsradien in der Ebene der } & -Achse \\
& & freie Außenspiegel & -Achse & $a$-Achse & $b$-Achse & $\begin{array}{c}\text { Temperatur- } \\
\text { erhöhung }\end{array}$ \\
\hline LG 55 & $\begin{array}{c}\text { Rotationsellipsoid } \\
\text { Elliptischer Zylinder }\end{array}$ & $\begin{array}{c}161 \mathrm{~m} \\
69 \mathrm{~m}\end{array}$ & $\begin{array}{c}144 \mathrm{~m} \\
184 \mathrm{~m}\end{array}$ & $\begin{array}{c}43,5 \mathrm{~m} \\
18,5 \mathrm{~m}\end{array}$ & $\begin{array}{c}39 \mathrm{~m} \\
50 \mathrm{~m}\end{array}$ & $\begin{array}{c}\left(0,69^{\circ} \mathrm{C}\right) \\
0,69\end{array}$ \\
\hline LG 52 & Elliptischer Zylinder & $168 \mathrm{~m}$ & $408 \mathrm{~m}$ & $84 \mathrm{~m}$ & $204 \mathrm{~m}$ & $0,36^{\circ} \mathrm{C}$ \\
\hline
\end{tabular}

Tab. 2. Kleinste bei Neodymglaslasern $(l=7 \mathrm{~cm})$ in verschiedenen Pumpanordnungen auftretende Krümmungsradien (Abb. 6) der Resonatorendflächen bei freien und auf den Stab aufgedampften Verspiegelungen sowie Temperaturerhöhung in der Stabachse (3). Die angegebenen Werte gelten am Schwellenwert. 
derung der optischen Weglänge proportional ist zur Energie, die im gleichen Volumelement aus der Pumpstrahlung absorbiert wurde. Um den Umfang der Rechnung erträglich zu halten, wird die Rechnung nur in zwei Dimensionen ausgeführt, es werden nur Strahlen berücksichtigt, die sich in einer Ebene senkrecht zur Zylinderachse ausbreiten. CookE et al. ${ }^{18}$ haben für den einfacheren Fall des Stabes im homogenen Strahlungsfeld gezeigt, daß die $\mathrm{Ab}$ weichungen einer Rechnung im Zweidimensionalen von einer genaueren Rechnung unter Einschluß der nicht im Hauptschnitt verlaufenden Strahlen 12\% nicht überschreiten. Die entsprechenden Abweichungen für den vorliegenden Fall sollten von der gleichen Größe sein. Da in der Ellipse das beleuchtende Strahlungsfeld nicht homogen ist, sind die Ergebnisse der Rechnung von Cooke et al. im übrigen hier nicht anwendbar.

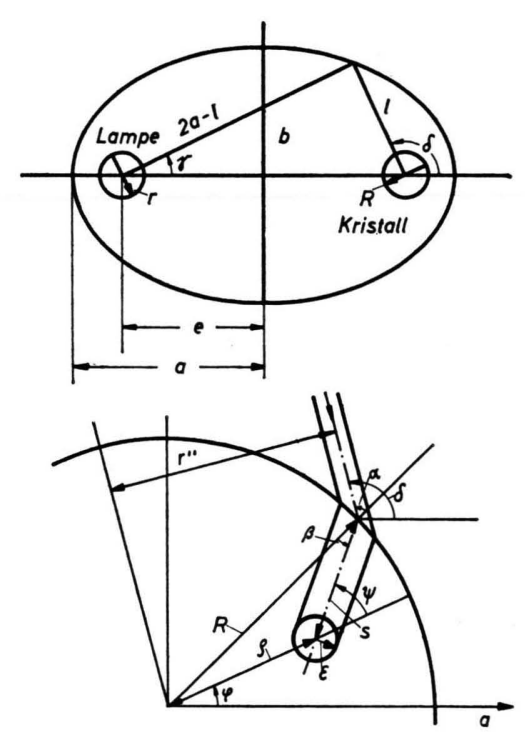

Abb. 7. Zur Berechnung der Dichte der absorbierten Energie im Laserstab. Oben: Lampe und Kristall im elliptischen Zylinder. Unten: Ausbreitung des Lichtes im Stab.

Die in der Rechnung auftretenden Bezeichnungen sind in Abb. 7 erläutert. Es werde ein kreisförmiges Volumelement im Laserstab betrachtet mit dem Radius $\varepsilon$ und der Höhe $\mathrm{d} h$, dessen Mittelpunkt durch die Polarkoordinaten $\varrho<R$ und $\varphi$ beschrieben wird. Um den Winkel $\psi$ geneigt gegen den Radiusvektor $\varrho$ falle ein Parallel-Bündel des Querschnittes $2 \varepsilon \cdot \mathrm{d} h$ auf das Volumelement. Die Stelle der Mantelfläche,

18 C. Н. Соoкe, J. McKenna u. J. G. Skinner, Appl. Opt. 3, 957 [1964]. die von dem Partialbündel durchsetzt wird, sei eben. Wir vernachlässigen also die Fokussierung dieses in den Kristall eintretenden parallelen Partialbündels, indem wir uns den kreisförmigen Querschnitt des Kristalls durch ein Polygon ersetzt denken. Wenn wir weiterhin annehmen, daß die Durchmesser von Lampe und Kristall $r$ und $R$ klein gegen die Ellipsenabmessungen $a, b$ und $e$ sind, dann kann man auch die Divergenz eines unter dem Winkel $\gamma$ von der Lampe ausgehenden Bündels vernachlässigen.

Wir betrachten die Blitzlampe als LAMBERTschen Strahler, dann können wir die Lampe durch eine ebene Fläche der Abmessungen $2 r \cdot \mathrm{d} h$ ersetzen, von der durch das senkrecht davon ausgehende Bündel nach der Reflexion an der Ellipse ein Bild $2 r^{\prime} \cdot \mathrm{d} h$ am Ort des Kristalls erzeugt wird. Wir erhalten folgende geometrische Beziehungen:

Aus der Gleichung für den Radiusvektor

folgt :

$$
l=b^{2} /(a+e \cos \delta)
$$

$$
\frac{r^{\prime}}{r}=\frac{l}{2 a-l}=\frac{b^{2}}{a^{2}+e^{2}+2 a e \cos \delta} .
$$

Mit dem Winkel $\psi$ sind die anderen Größen verknüpft durch:

$$
\sin \beta=(\varrho / R) \sin \psi(5 \mathrm{a}) ; \quad \sin \alpha=n(\varrho / R) \sin \psi(5 \mathrm{~b})
$$

$\delta=\varphi+\psi+\alpha-\beta$

$$
r^{\prime \prime}=n \varrho \sin \psi \text {. }
$$

Für die Strecke $s$, die das Bündel vom Kristallrand bis zum Volumelement $\varrho, \varphi$ zurückzulegen hat, erhält man:

$$
s=\sqrt{R^{2}-Q^{2} \sin ^{2} \psi}-\varrho \cos \psi .
$$

Für die Strecke $d$, die ein Bündel bei der vollständigen Durchquerung des Stabes zurückzulegen hat, ergibt sich:

$$
d=2 R \cos \beta .
$$

Zur Berechnung der im Volumelement absorbierten Leistung benutzen wir die Tatsache, daß bei verlustfreier Abbildung, die wir voraussetzen wollen, das abbildende Element mit der gleichen Strahlungsdichte $S_{0}{ }^{*}$ wie der Sender leuchtet. Lassen wir zunächst die Strahlen, die den Kristall nach Reflexion an der Grenzfläche mehrfach durchlaufen, außer acht, so ergibt sich für die in das betrachtete Teilbündel abgestrahlte spektrale Leistung $\mathrm{d} L(v)$ :

$$
\mathrm{d} L(v)=S_{0}{ }^{*}(v) \mathrm{d} v \cdot \mathrm{d} \delta \cdot \mathrm{d} r^{\prime} \cdot \mathrm{d} h .
$$

Für $\mathrm{d} \delta$ folgt aus (6) :

$\mathrm{d} \delta=\mathrm{d} \psi\left[1+\frac{\varrho \cos \psi}{R \cos \alpha \cos \beta}(n \cos \beta-\cos \alpha)\right]$. 
Beim Eintritt des Lichtes in den Kristall wird seine Strahlungsdichte um den Faktor $n t^{2}$ geschwächt. Wir nehmen unpolarisiertes Licht an. Nach den Fresnelschen Formeln ergibt sich:

$$
t^{2}=\frac{1}{2}\left(t_{\|}^{2}+t_{\perp}^{2}\right)=\frac{2 \cos ^{2} \alpha}{(n \cos \beta+\cos \alpha)^{2}}\left[\frac{1}{\cos ^{2}(\alpha-\beta)}+1\right] .
$$

Nach (10), (11) und (12) ist demnach die Leistung, die das Bündel mit dem Querschnitt $2 \varepsilon \cdot \mathrm{d} h$ nach dem Eintritt in den Stab führt, gegeben durch

$\mathrm{d} L(\nu, \psi)=S_{0}{ }^{*}(v) \mathrm{d} \nu \cdot 4 n \varepsilon \cdot \mathrm{d} h \frac{\cos ^{2} \alpha}{(n \cos \beta+\cos \alpha)^{2}} \cdot\left(1+\frac{1}{\cos ^{2}(\alpha-\beta)}\right)\left[1+\frac{\varrho \cos \psi}{R \cos \alpha \cos \beta}(n \cos \beta-\cos \alpha)\right] \mathrm{d} \psi$.

In (13) ist der Betrag von $\mathrm{d} \delta$ einzusetzen, weil der Beitrag des Partialbündels $\mathrm{d} \delta$ zu $\mathrm{d} L(\nu, \psi)$ immer positiv sein muß. Die aus diesem Bündel in dem kreisförmigen Volumelement absorbierte Leistung ist:

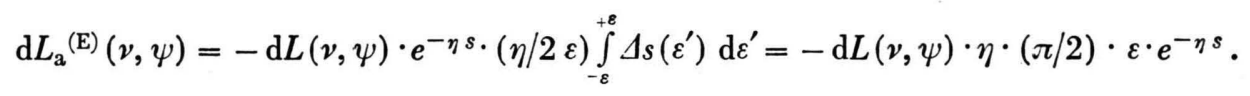

Wegen $\mathrm{d} V=\pi \varepsilon^{2} \mathrm{~d} h$ ergibt sich für die Dichte der absorbierten Leistung $\mathrm{d} W_{\mathrm{a}}{ }^{(\mathrm{E})}(v, \psi)$ bei einmaligem (Index E) Durchgang der Pumpstrahlung durch den Stab

$$
\mathrm{d} W_{\mathrm{a}}{ }^{(\mathrm{E})}(\nu, \psi)=\mathrm{d} L_{\mathrm{a}}{ }^{(\mathrm{E})}(\nu, \psi) / \mathrm{d} V=-[\mathrm{d} L(\nu, \psi) / \mathrm{d} h] \cdot(\eta / 2 \varepsilon) \cdot e^{-\eta s} .
$$

Berücksichtigen wir jetzt die $z$-fachen Mehrfachreflexionen (Index M) im Stab, so erhalten wir mit

$$
r_{\perp}^{2}=\{(n \cos \beta-\cos \alpha) /(n \cos \beta+\cos \alpha)\} ; \quad r \|^{2}=r_{\perp}^{2} \cdot\left[\cos ^{2}(\alpha+\beta)\right] /\left[\cos ^{2}(\alpha-\beta)\right]
$$

(Reflexionsvermögen der Grenzfläche Stab-Luft) und $\delta_{z}=\varphi+\psi+\alpha-(2 z+1) \beta+z \pi$ :

$$
\begin{array}{r}
\mathrm{d} W_{\mathrm{a}}{ }^{(\mathrm{M})}(v, \psi)=2 n \frac{\cos ^{2} \alpha}{(\cos \alpha+n \cos \beta)^{2}} \sum_{z=0}^{\infty}\left[1+\frac{\varrho \cos \psi}{R \cos \alpha \cos \beta}(n \cos \beta-(2 z+1) \cos \alpha)\right] \\
\cdot\left\{\frac{1}{\cos ^{2}(\alpha-\beta)} \cdot r^{2 z}+r_{\perp}^{2 z}\right\} \cdot S_{0}{ }^{*} \eta \cdot \exp \{-\eta(s+z d)\} \mathrm{d} \nu \mathrm{d} \psi .
\end{array}
$$

In dieser Summe ist das erste Glied (mit $z=0$ ) identisch mit $\mathrm{d} W_{\mathrm{a}}^{(\mathrm{E})}(v, \psi)$. Die Summation der geometrischen und abgeleiteten Reihen liefert:

$$
\begin{gathered}
\mathrm{d} W_{\mathrm{a}}{ }^{(\mathrm{M})}(v, \psi)=\mathrm{d} W_{\mathrm{a}}{ }^{(\mathrm{E})}(\nu, \psi)+\frac{2 n \cos \alpha}{\cos \beta(\cos \alpha+n \cos \beta)^{2}} \cdot S_{0}{ }^{*} \eta \cdot e^{-\eta s} \mid\left\{\left[\cos \alpha \cos \beta+\frac{\varrho}{R} \cos \psi(n \cos \beta-\cos \alpha)\right]\right. \\
\cdot\left(\frac{1}{\cos ^{2}(\alpha-\beta) \cdot\left(r_{\|}^{-2} e^{\eta} d-1\right)}+\frac{1}{r_{\perp}^{-2} e^{\eta} d-1}\right)-\frac{2 \varrho}{R} \cos \psi \cos \alpha \\
\left.\cdot\left(\frac{r_{\|}^{-2} e^{\eta} d}{\cos ^{2}(\alpha-\beta) \cdot\left(r_{\|}^{-2} e^{\eta} d+1\right)^{2}}+\frac{r_{\perp}^{-2} e^{\eta} d}{\left(r_{\perp}^{-2} e^{\eta} d-1\right)^{2}}\right)\right\} \mid \mathrm{d} v \mathrm{~d} \psi .
\end{gathered}
$$

Zur Berechnung der gesamten Energiedichte ist noch die Integration über $v$ und $\psi$ auszuführen:

$$
W_{\mathrm{a}}{ }^{(\mathrm{M})}=\int_{\nu=0}^{2 \pi} \int_{\nu=0}^{\infty} \mathrm{d} W_{\mathrm{a}}{ }^{(\mathrm{M})}(\nu, \psi) \mathrm{d} \nu \mathrm{d} \psi .
$$

Die Inhomogenität des Strahlungsfeldes um den Kristall im elliptischen Zylinder ist in (18) noch nicht enthalten, sie tritt erst bei der Ausführung der Integration über $\psi$ in der Form von einschränkenden Bedingungen über den Integrationsbereich von $\psi$ in Erscheinung [Strich am Integralzeichen in (18)] und hat die folgenden drei Ursachen:

1. Ein Teil des elliptischen Zylinders empfängt von der Lampe kein Licht, sondern liegt im Schatten des Kristalls. Die entsprechenden Winkel $\delta$ liegen um die $a$-Achse und sind näherungsweise gegeben durch $\delta_{1,2}= \pm \frac{1}{2}(R / e) \cdot(a+e) /(a-e)$.

2. In bestimmten Bereichen des Einfallwinkels $\delta(\psi)$ ist die Breite des Lampenbildes $2 r^{\prime}$ am Kristallort kleiner als der Kristalldurchmesser $2 R$. Die Randzonen des Laserstabes werden also nicht aus allen Richtungen her, d. h. von der gesamten Fläche des elliptischen Zylinders beleuchtet.

3. Für bestimmte $\psi$-Werte kann der Winkel $\beta$ nach (5 a) größer als der Grenzwinkel der Totalreflexion werden. Für solche $\psi$-Werte muß der Integrand verschwinden. Bedingung 3 betrifft wie 2 nur die Randzonen des Kristalls, die Licht nur aus bestimmten Teilbereichen von $\psi$ empfangen, 
deren Größe für wachsendes $\varrho$ ständig abnimmt. In achsennahen Bereichen ist dagegen, abgesehen von Einschränkung 1, die Ausleuchtung homogen ${ }^{19}$.

Zur vollständigen Berücksichtigung dieser drei Bedingungen müßte jedes Glied der Reihe (16) einzeln geprüft werden und der Beitrag solcher Glieder, die unter wenigstens eine Einschränkung fallen, gleich Null gesetzt werden. Damit ist aber eine geschlossene Summation der Reihen nicht mehr möglich, wir haben deswegen die strenge Berücksichtigung aller Einschränkungen nur bei der Berechnung des ersten Gliedes $W_{\mathrm{a}}{ }^{(\mathrm{E})}$ durchgeführt, das ja den Hauptbeitrag zur Dichte der absorbierten Energie liefert, und bei der Berechnung des Beitrags der mehrfach reflektierten Strahlen nur Bedingung 3 berücksichtigt. Dadurch fällt die so berechnete Energiedichte $W_{\mathrm{a}}{ }^{(\mathrm{M})}$ in den Randzonen des Kristalls etwas höher aus als bei exakter Rechnung. Da die Beiträge der Mehrfachreflexion aber, wie die Ergebnisse zeigen, nur eine kleine Korrektur liefern (Abb. 8), ist diese Vernachlässigung ohne Bedeutung.
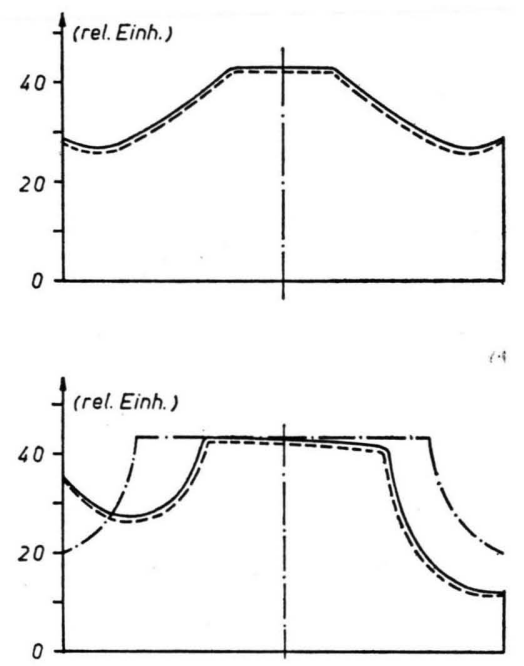

Abb. 8. Berechnete Verteilung der Dichte der absorbierten Energie (relative Einheiten) in einem LG 55-Stab im elliptischen Zylinder (Lichtausbreitung nur in der Hauptschnittebene). Oben: Schnittebene parallel zur kleinen Ellipsenachse. Unten: Schnittebene parallel zur großen Achse. Ausgezogene Kurven: Energieverteilung unter Einschluß der Mehrfachreflexionen im Kristall. Gestrichelte Kurve: Energieverteilung ohne Mehrfachreflexionen. Strichpunktierte Kurve: Energiedichte in einem nicht absorbierenden Stab im isotropen Strahlungsfeld nach ${ }^{17}$ (zum Vergleich). Diese Kurve wurde an die Dichte im Achsenpunkt des absorbierenden Stabes angepaßt.
Zur maschinellen Berechnung von Zahlenwerten für $W_{\mathrm{a}}{ }^{(\mathrm{E})}$ und $W_{\mathrm{a}}{ }^{(\mathrm{M})}$ mit der Simpsonschen Regel für vorgegebene Werte von $\varrho$ und $\varphi$ wurden die Absorptionskonstanten $\eta(v)$ beider Neodymglassorten aus Angaben des Herstellers bestimmt, für den Verlauf der Strahlungsdichte $S_{0}{ }^{*}(v)$ der Xenonblitzlampe wurden die Angaben von Marshak ${ }^{20}$ benutzt. Die Ergebnisse für LG 55 in einer Pumpellipse mit den oben angegebenen Abmessungen sind in Abb. 8 dargestellt in Form zweier Schnitte durch den Stabquerschnitt parallel zur großen und kleinen Ellipsenachse. Außerdem ist die nach ${ }^{17}$ berechnete Verteilung der Energiedichte in einem nicht absorbierenden Stab mit gleichem Brechungsindex im homogenen Beleuchtungsfeld eingezeichnet. Man erkennt, $\mathrm{da} ß$ in der Ellipse der angenähert homogen ausgeleuchtete Bereich kleiner ist und ovalen Querschnitt hat als Folge der abbildenden Eigenschaften des elliptischen Zylinders. Ferner ist im Schnitt parallel zur großen Ellipsenachse die stärkere Konzentration der Pumpcnergie auf der der Lampe zugekehrten linken Seite und damit die Neigung der Stabendflächen deutlich.

Bei einem Vergleich der gemessenen (Abb. 6) und der gerechneten Kurven ist zunächst der Vernachlässigung der schräg zum Hauptschnitt laufenden Pumpstrahlung Rechnung zu tragen. Die Ergebnisse bei Berücksichtigung dieser Strahlung im einfacheren Fall der homogenen Verteilung der Pumpenergie ${ }^{17}$ legen die Vermutung nahe, daß auch hier die vollständigere Rechnung eine Abrundung des Kurvenverlaufs bringen wird im Einklang mit den Beobachtungen (Abb. 6), ohne aber an der unterschiedlichen Größe der Energiekonzentration und damit der $\mathrm{Zu}$ nahme der optischen Weglänge in den einzelnen Bereichen wesentliches zu ändern. Diese Differenzen erscheinen in den gerechneten Kurven deutlicher als in den gemessenen. Für diese Glättung des experimentellen Kurvenverlaufs sind zwei Ursachen verantwortlich:

1. Die Politur der Mantelflächen der Laserstäbe ist meist nicht so vollkommen, daß nicht eine merkliche Streuung der Pumpstrahlung beim Eintritt in den Kristall und den erneuten inneren Reflexionen eintreten würde.

2. Gewisse Abweichungen von der exakten Form eines elliptischen Zylinders sind bei dessen technischer Realisation unvermeidbar und führen zu

20 J. S. MarshaK, Appl. Opt. 2, 793 [1963]. 
einer unvollkommenen Bündelung der Pumpstrahlung im Kern des Laserstabes.

Die in der Rechnung hervortretende Aufwölbung der Randzonen des Kristalls, die dort durch das starke Anwachsen der Absorptionskonstante des Glases im nahen UV verursacht wird, ist in unseren Aufnahmen nur in einigen Fällen bei starkem Pumpen am Ende des Pumpimpulses erkennbar. Diese Diskrepanz geht zum Teil darauf zurück, daß die in unseren Experimenten benutzten Blitzlampen nicht mit so hohen Leistungen betrieben wurden wie die in ${ }^{20}$ verwendeten und daß damit der UV-Anteil der
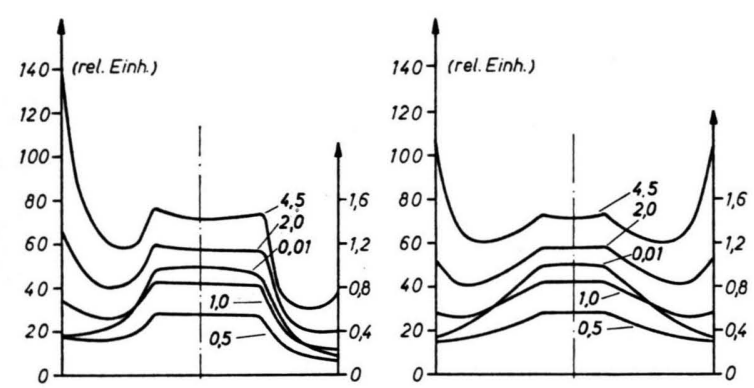

Abb. 9. Berechnete Verteilung der absorbierten Energie im Neodymglasstab bei variablem Absorptionsvermögen des Stabes. Links: Schnitt parallel zur großen Achse $a$ des Pumpellipsoids. Rechts: Schnitt parallel zur kleinen Achse $b$ des Ellipsoids. Die Zahlen an den Kurven geben die Faktoren an, mit denen das Absorptionsvermögen $\eta(v)$ von LG 55 multipliziert wurde. Der Ordinatenmaßstab für die Kurve $0,01 \cdot \eta(v)$ ist um den Faktor 50 erhöht und rechts aufgetragen.
Lampen-Emission hier kleiner war. Außerdem wird die UV-Strahlung an kleinen Unregelmäßigkeiten der Verspiegelung besonders stark gestreut und deshalb schlecht auf dem Stab gebündelt.

Zum Studium der Abhängigkeit der Dichte der absorbierten Energie von der Konzentration der Neodymionen in der Glasmatrix wurde die Funktion $\eta(v)$ mit einem Faktor zwischen 0,01 und 5 multipliziert und die Energieverteilung berechnet. Ergebnisse sind in Abb. 9 aufgetragen. Man erkennt das starke Anwachsen der Aufwölbung der Randzonen des Stabes mit steigender Konzentration und damit eine Umkehrung des Krümmungssinns der Endflächen. Entsprechende Rechnungen für LG 52 zeigen wegen des stärkeren Absorptionsvermögens dieses Glases im nahen UV eine stärkere Abrundung der Kurven im Einklang mit den experimentellen Beobachtungen (Abb. 6).

Wir möchten Herrn Prof. Dr. K. H. Hellwege für die kritische Durchsicht des Manuskripts danken. Weiterhin sind wir Herrn Dr. N. Neuroth von den Jenaer Glaswerken S ch ot t u. G e n., Mainz, für geeignete Neodymglas-Proben sowie Herrn Dr. D. Röss von dem Zentrallaboratorium der Siemens \& $\mathrm{Halske} \mathrm{AG,}$ München, für die Überlassung eines der von ihm entwickelten Rotationsellipsoide zum Pumpen von KristallLasern dankbar. Unser Dank gilt ferner dem Rechenzentrum der Technischen Hochschule Darmstadt für die Bereitstellung von Maschinenzeit und der Deutschen Forschungsgemeinschaft für die finanzielle Unterstützung dieser Untersuchung. 